\title{
Evolución en los conocimientos sobre alimentación: una intervención educativa en estudiantes universitarios
}

\author{
Developments in food knowledge: \\ an educational intervention \\ in university studentes
}

\begin{abstract}
To analyze the relationship between the level of knowledge about food, the degree of adherence to the Mediterranean Diet (MeDi) and nutritional status; an intervention study was performed with pre- and post-intervention evaluation after three educational sessions, designed according to the learning preferences of health science university students. Sixty-five physiotherapy and podology students participated. The mean age was 22.0 years and participants were mostly male $58.5 \%$. The majority were normal weight $(63.1 \%)$, but many $(69.2 \%)$ needed to improve their eating habits in order to comply with DiMe recommendations. The statistical relationship found between the level of previous knowledge in nutrition and nutritional status (Pearson's Correlation Coefficient $=0.7 ; p=0.049$ ) suggests that nutritional education actions in this group could contribute to improving the quality of the diet, nutritional status and future health of university students. Educational sessions were able to improve the level of knowledge regarding nutrition and food in the majority of students, thus it is possible to consider the intervention as an efficient tool for the transmission of nutritional knowledge in this group. Key words: Food habits; Nutritional education; Mediterranean diet; Learning strategies.
\end{abstract}

\section{INTRODUCCIÓN}

La Dieta Mediterránea (DiMe) está considerada como un modelo dietético saludable, relacionado con una mayor expectativa de vida y con una reducción de la incidencia de enfermedades crónicas no trasmisibles (1-3). No obstante, en los últimos años se han experimentado cambios en el patrón dietético tradicional de la DiMe (4), justificados por un contexto sociocultural que influye en la organización familiar, condicionando el tiempo que dedican las familias a la selección y preparación de los alimentos y provocando un aumento en la preferencia de alimentos procesados (5-7).

Entre los grupos de riesgo que incurren en el distanciamiento del patrón de la DiMe, destacan los adolescentes y los jóvenes universitarios. Estudios que analizan este fenómeno social, proponen que los cambios en los patrones de alimentación se relacionan con un aumento de la tendencia al sobrepeso y a la obesidad $(4,8)$, favorecidos por el abandono
Violeida Sánchez (1)

Alicia Aguilar (2)

Fabián González (1)

Laura Esquius (1)

Cristina Vaqué (3)

(1) Facultad de Ciencias de la Salud de Manresa, Universidad de Vic-Universidad Central de Cataluña (UVic-UCC), Barcelona, España. (2) Departamento de Ciencias de la Salud. Universitat Oberta de Catalunya (UOC), España. (3) Facultad de Ciencias de la Salud y el Bienestar. Universidad de Vic-Universidad Central de Cataluña (UVic-UCC), España.

Dirigir la correspondencia a: Violeida Sánchez Socarrás Facultad de Ciencias de la Salud de Manresa, Universidad de Vic-Universidad Central de Cataluña (UVic-UCC), Barcelona

Teléfono 938774179 Email: vsanchez@umanresa.cat

Este trabajo fue recibido el 21 de Marzo de 2016 y aceptado para ser publicado el 23 de Diciembre de 2016.

de los hábitos alimentarios familiares, por factores estéticos o por la influencia del entorno social $(1,9)$.

Algunos investigadores destacan la necesidad de realizar actividades educativas e implementar programas de intervención nutricional, que eleven el nivel de conocimientos acerca de las características y beneficios de la DiMe, como un modelo alimentario que podría mejorar las expectativas de salud en la edad adulta $(1,9)$.

Que las acciones de educación nutricional se adapten a las necesidades y a las características de los receptores, es una de las premisas para garantizar su calidad (10). Si asumimos que la educación nutricional es un proceso de aprendizaje e interiorización de habilidades, hábitos y conductas; esta adaptación podría beneficiarse del empleo de técnicas educativas afines a la manera de aprender de los participantes (10-12), sobre la base de estas consideraciones se diseñó la presente investigación, que tuvo como objetivos: 1) analizar la relación 
entre el nivel de conocimientos en alimentación, el grado de adherencia a la DiMe y el estado nutricional de un grupo de estudiantes universitarios y 2) evaluar el impacto en el nivel de conocimientos en alimentación de una intervención educativa, diseñada según las preferencias de aprendizaje de los participantes.

\section{MATERIAL Y MÉTODOS}

De septiembre de 2013 a enero de 2015 se realizó en la Facultad de Ciencias de la Salud de Manresa en Barcelona, un estudio de intervención con evaluación pre y post-intervención. Participaron universitarios matriculados en los estudios de Grado en Fisioterapia y Podología.
Instrumentos de evaluación

1) Cuestionario validado sobre conocimientos en nutrición y alimentación. Para evaluar el nivel de conocimientos de los estudiantes antes y una semana después de realizada la intervención educativa. Consta de 27 preguntas distribuidas en cuatro categorías: hábitos alimentarios, estado nutricional, alimentos y nutrientes y alteraciones o procesos relacionados con la alimentación. La calificación global es de 0 a 100 puntos, considerando $<50$ puntos un nivel de conocimientos bajo, entre 50,0 y 69,9 un nivel medio $y \geq 70$ puntos un nivel elevado (anexo 1). La escala fue elaborada y validada por los autores de este estudio. En el proceso de validación participaron 158 estudiantes

\section{ANEXO 1}

Cuestionario de conocimientos generales en nutrición y alimentación, en estudiantes universitarios de Ciencias de la Salud.

\section{Orientaciones para responder:}

- Cada pregunta tiene sólo una opción de respuesta correcta.

-Las preguntas con más de una respuesta seleccionada serán anuladas.

-No responder a una pregunta, se interpretará como que el encuestado desconoce la respuesta.

\section{Hábitos alimentarios}

1.- ¿Cuántas veces recomiendan los expertos que se ha de comer al día?
O Tres
O Cinco
O De tres a cinco veces
Siempre que sienta hambre

2.- ¿Cuánta agua se recomienda beber durante el día?
O Menos de un litro
- Más de dos litros
- Cerca del $10 \%$ de las calorías
- Alrededor del 15\% de las calorías
O Entre el $20-25 \%$ de las calorías
○ El 25\% o más de las calorías

- Entre uno y dos litros, sea invierno o verano

O Entre uno y dos litros, pero sólo en verano

3.- El desayuno ha de aportar a la dieta diaria:

4.- Un desayuno equilibrado ha de contener:

O Pan, lácteos y alimentos ricos en proteínas

○ Lácteos, frutas y cereales

O Pan, embutidos y lácteos

Lácteos, frutas y alimentos ricos en proteínas

5.- ¿Cuántas raciones de frutas y vegetales, recomiendan los expertos que se han de ingerir al día?

$\bigcirc$ Una de fruta y una de verdura

Una de fruta y tres de verduras

Tres de frutas y dos de verduras

Tres de verduras y tres o más de frutas

\section{Estado nutricional}

6.- El índice de masa corporal (IMC):

- En las personas con obesidad varía de 25 a 30

○ Permite estimar el grado de obesidad

- Permite calcular la composición corporal

- Si es inferior a 18,5 la persona está en normo peso

7.- Una persona tiene sobrepeso sí:

- No tiene el peso ideal para su altura

- Si su IMC es superior a 30

○ Si su IMC está entre 25 y 30

Si su IMC está entre 18 y 20 
8.- Las necesidades energéticas de una persona dependen:

O Sólo del peso y la altura

De su edad

O Sólo de la actividad física que realiza diariamente

- Del peso, la altura, la edad y la actividad física

9.- El consumo energético diario indica la energía consumida:

O Para mantener la temperatura corporal a $37^{\circ}$

- Durante el día

- Durante el día, exceptuando las horas de sueño

Durante la práctica de ejercicio físico

10.- ¿Cuál opción sería más recomendada para perder peso?

○ Dieta hipoglucémica y ejercicio físico

- Dieta hiperproteíca indicada por un profesional de la salud y ejercicio físico

- Dieta hipoproteíca y ejercicio físico

- Dieta hipocalórica equilibrada y ejercicio físico

11.- La operación bikini:

O Es una opción adecuada para perder peso en los meses previos al verano

$\bigcirc$ Es una opción recomendada siempre que se acompañe de una dieta hipoglucémica

- No produce efecto rebote, siempre que se acompañe de ejercicio físico

- Puede producir un efecto rebote en el que se recupere el peso inicial

\section{III-Alimentos y nutrientes}

12.- ¿Cuál de los siguientes pescados es azul?

Merluza (lluç)

Bacalao (bacallà)

- Rape (rap)

O Salmón (salmó)

13.- ¿Cuál de los siguientes alimentos tiene un alto contenido en vitamina C?
El pan integral
L Los pimientos (pebrots)
El pescado azul
El aceite de oliva

14.- Respecto a las vitaminas es cierto que:

$\bigcirc$ Hay que ingerir la mayor cantidad posible

$\bigcirc$ Ingeridas en grandes cantidades pueden provocar efectos perjudiciales

- Cuando su ingesta es baja, las producimos de manera endógena

- Las hidrosolubles, las podemos adquirir a través del consumo de agua embotellada

15.- ¿Cuáles son las grasas que los expertos recomiendan no ingerir en exceso?
O Grasas mono-insaturadas
- Grasas poli-insaturadas
Grasas saturadas
Grasas vegetales

16.- ¿Qué caracteriza a la dieta mediterránea?

Consumo diario de carne

- Elevado consumo de frutas y verduras

- Elevado consumo de lácteos y moderado de grasas saturadas

O Consumo diario de aceite de oliva y vino

17.- Los transgénicos, son alimentos:

Importados de países extranjeros

$\bigcirc$ Que pueden incluir diferentes fragmentos de ADN de diferentes organismos

$\bigcirc$ Que no contienen gérmenes potencialmente patógenos

Obtenidos artificialmente y que no contienen sustancias tóxicas

18.- ¿Por qué la fibra alimentaria es importante en la dieta?

$\bigcirc$ Porque incrementa la digestibilidad de los alimentos

- Porque facilita el tránsito intestinal

O Porque libera agua en el intestino

$\bigcirc$ Porque aumenta la absorción de los nutrientes

19.- ¿Qué beneficios tienen los alimentos integrales?

O Son bajos en calorías

O Son bajos en azúcar

- Son bajos en sal

- Son ricos en fibra alimentaria 
IV-Alteraciones de la salud relacionadas con la alimentación y la nutrición.

20.- Los individuos alcohólicos:

Pueden presentar carencias de vitaminas y minerales

- Suelen llevar una dieta equilibrada

O Suelen acumular proteínas en el hígado

- Pueden absorber mejor los nutrientes

21.- ¿Cuáles de las alteraciones siguientes son Trastornos del Comportamiento Alimentario (TCA)?

O Obesidad y dislipemia (alteración del colesterol y/o triglicéridos)

- Anorexia nerviosa y Bulimia nerviosa

- Enfermedad celíaca y dispepsia

O Todas las alteraciones anteriores son TCA

22.- En un individuo adulto con hiperlipemia (aumento del colesterol y/o triglicéridos), es recomendable:

○ Un consumo elevado de azúcares simples (azúcar, miel, zumo de frutas, etc.)

O Una dieta acorde a sus necesidades energéticas o ligeramente inferior

- Aumentar el consumo de carnes rojas

O Consumir leche entera en lugar de desnatada

23.- ¿Cuál de los siguientes factores, NO predispone a padecer osteoporosis (descalcificación de los huesos)?

O La menopausia

$\bigcirc$ Una ingesta insuficiente de calcio en la dieta

El consumo de alimentos ricos en vitaminas A, B y ácido fólico

$\bigcirc$ El consumo excesivo de bebidas ricas en cafeína

24.- ¿Qué alimento se recomienda evitar en caso de hipertensión arterial?
O Aceite de oliva
O Patatas
Caldos y sopas concentrados
O Frutas frescas

25.- La Diabetes Mellitus:

O Es causada por alteraciones en el metabolismo de lípidos y glúcidos complejos

O Puede deberse a alteraciones en la calidad y cantidad de la insulina

- Sólo la padecen adultos mayores de 40 años

- Aparece por consumir alimentos dulces diariamente

26.- Una intoxicación alimentaria es una enfermedad causada por:

O Consumo excesivo de alimentos que contienen vitaminas y minerales

- Consumo de alimentos contaminados

O Consumo excesivo de alimentos muy condimentados

C Consumo de alimentos transgénicos

27.- ¿Por qué no es recomendable en un deporte aeróbico consumir azúcar en la hora previa a la competición o entrenamiento?

O Porque la deshidratación asociada al ejercicio favorece la aparición de vértigo

O Porque se estimula la liberación de insulina que es inhibidora de la lipólisis

- Porque su digestión es lenta y hace que la práctica deportiva suponga un esfuerzo mayor

○ Al contrario, sí es recomendable porque el azúcar aporta energía

universitarios, con edades comprendidas entre los 18 y 50 años con un promedio de edad de $20,8 \pm 5,1$ años. El $45,5 \%$ fueron mujeres; procedentes de la Facultad de Ciencias de la Salud de Manresa, Barcelona. El proceso de validación se realizó por método de test-retest, con aplicaciones sucesivas auto-administradas del cuestionario en diferentes etapas. Los valores obtenidos en el cálculo de la consistencia interna para cada ítem y para el conjunto, la correlación ítem global y el grado de estabilidad temporal encontrados, garantizan que la escala validada pueda ser utilizada con garantían en la evaluación del nivel de conocimientos en nutrición y alimentación.

2) Cuestionario VARK (Visual, Auditivo, Lecto-escritor y Kinestésico) $(13,14)$ para identificar los Estilos de Aprendizaje (EA) de los estudiantes y seleccionar las estrategias de aprendizaje preferidas por la mayor parte del colectivo. Según estas estrategias se seleccionaron las técnicas de enseñanza a incluir en el diseño de las sesiones educativas.

3) Cuestionario KidMed que permitió evaluar el grado de adherencia de los universitarios a la DiMe, y si existía correlación de este con el estado nutricional y con el nivel de conocimientos iníciales en nutrición demostrados por los encuestados. Según los resultados del test KidMed (15), una puntuación $\leq 3$ indica baja adhesión, entre 4 y 7 una adhesión media y una puntuación total $\geq 8$ indica una dieta de alta adhesión a la DiMe $(1-3,15)$.

Los tres cuestionarios se aplicaron en forma de encuesta auto administrada y con la autorización del Decanato de la Facultad de Ciencias de la Salud de Manresa y las subdirecciones docentes de los estudios implicados. Los estudiantes fueron informados de que participaban en una investigación científica y se garantizó respetar el anonimato de los datos personales solicitados en los encabezamientos de los cuestionarios (edad, sexo y estudios matriculados).

La presente investigación forma parte del trabajo de campo para la tesis doctoral de uno de los autores del artículo; de ahí que fuera aprobada por la Comisión Científica del Programa de Doctorado en Salud y Calidad de Vida de la UVic-UCC. 
Intervención educativa

Se realizaron tres sesiones educativas que incluyeron las actividades relacionadas en la tabla 1. En las sesiones educativas se utilizaron recursos y materiales de aprendizaje adaptados a las estrategias de aprendizaje preferidas por la mayoría de los participantes (estudiantes con EA auditivo y kinestésico). En la sesión 1 se analizaron las pautas de alimentación que caracterizan la DiMe. En la sesión 2 se confeccionó un puzle con un listado de órganos, alimentos y nutrientes, que intervienen en la fisiopatología de algunas alteraciones de la salud, que pueden manifestarse en individuos con un modelo de alimentación poco saludable, haciendo énfasis en los beneficios que podría aportar la DiMe en estas alteraciones. En la sesión 3 se proyectaron imágenes con referencias gráficas explicitas de alteraciones y procesos relacionados con la alimentación (anorexia nerviosa, obesidad, episodios de purgas, actividad física, control de la tensión arterial e ingesta de vino con las comidas), a partir de las imágenes se realizó una lluvia de ideas que sirvió como guía para debatir sobre alteraciones de la salud relacionadas con la alimentación.

\section{ANÁLISIS ESTADÍSTICO}

Las respuestas se analizaron mediante el paquete estadístico SPSS Versión 15.0 para Windows. En el análisis descriptivo de los datos se expresaron las variables cuantitativas como medias y desviaciones estándar, las variables cualitativas se describieron mediante frecuencias absolutas y relativas. El test chi-cuadrado permitió contrastar las proporciones entre las variables cualitativas (porcentaje de respuestas a los ítems del test KidMed y relación de dependencia de los EA con el nivel de conocimientos adquiridos).
El estado nutricional se estimó del cálculo del IMC a partir del peso y altura referidos. Para el análisis del nivel de conocimientos previos y adquiridos, se tuvo en cuenta la calificación media obtenida en el global del cuestionario y en las categorías que lo integran.

En la interpretación de las respuestas del cuestionario VARK se consideró que un estilo era dominante cuando fue elegido en más del $50 \%$ de las respuestas y si dos o más estilos alcanzan este porcentaje, la categoría correspondería a un estilo bimodal $(B M=$ combinación de dos preferencias dominantes) o multimodal $(M M=$ combinación de tres $\mathrm{O}$ más preferencias dominantes). En caso de que ningún estilo obtuviera el $50 \%$, el umbral de aceptación se bajó al $40 \%$ (preferencia fuerte) $(13,14)$.

Se utilizaron tablas de contingencia para explorar la relación de dependencia entre las variables, para las cuantitativas (estado nutricional según valor del IMC, adherencia a la DiMe según valor del índice KidMed y nivel de conocimientos según la calificación media obtenida), se calculó el Coeficiente de Correlación de Pearson (CCP) y para las cualitativas la $\vee$ de Cramer. Los valores del $C C P \geq 0,7$ fueron considerados indicadores de buena correlación. Para todos los cálculos se consideró un nivel de confianza del 95\%.

\section{RESULTADOS}

Las características de los 65 estudiantes que participaron en el estudio se resumen en la tabla 2 . En lo que respecta al grado de adherencia a la DiMe no se evidenciaron diferencias significativas entre ambos sexos $(6,18 \pm 2,25$ en las mujeres $y$ $6,42 \pm 2,45$ en hombres; (Coeficiente de contingencia $V$ de Cramer $=0,7 ; p=0,420$ ). Los estudiantes con EA de tipos $B M$

TABLA 1

Técnicas de enseñanza empleadas según las estrategias de aprendizaje afines a los estilos de aprendizaje (EA) preferentemente identificados. (DiMe: Dieta Mediterránea).

\section{Tema a tratar}

Sesión 1. DiMe, hábitos alimentarios y estilo de vida

\section{Técnica de enseñanza}

Lectura guiada: "Hábitos alimentarios y conductas relacionadas con la salud en una población universitaria"16

Juegos de rol

Lectura comentada: "Características morfo-funcionales del aparato digestivo"

Puzle

Dinámica de estudio de casos:

"Pacientes con obesidad, dolor abdominal y alimentación poco saludable: asociado a lumbalgia y/o asimetría pélvica"
Estrategia de aprendizaje preferente

Lectura de fragmentos en voz alta y discusión del contenido

Desarrollar ejemplos y participar en situaciones que involucren experiencia y práctica

Auditivo

Actividad de aprendizaje cooperativo en grupos

Dinámica de grupo y discusión colectiva

\section{EA}

Auditivo
Instrucciones verbales

Kinestésico tricional estado relacionados con la alimentación
Lluvia de ideas
Propuestas, debate y discusión
Auditivo / Kinestésico 
y $M M$ manifestaron combinaciones de los estilos predominantes (auditivo y kinestésico) con las modalidades Lecto escritor y Visual.

El análisis de las respuestas al KidMed, reveló diferencias significativas respecto al consumo de algunos productos. Se observó que los varones dicen consumir más pescado, pasta, arroz y suelen desayunar cereales. Por su parte las mujeres refieren consumir más frutas, verduras y golosinas. Mientras que el consumo referido de frutos secos, aceite de oliva o lácteos, resultó similar en ambos sexos.

En la tabla 3 se muestra el nivel de conocimientos demostrados por los universitarios a través de las respuestas en las dos aplicaciones del cuestionario: Q1 (inicial) y Q2 (una semana después de las sesiones educativas).

Según los datos de la figura 1, la mayor parte de los estu- diantes demostró un nivel medio de conocimientos en las dos aplicaciones del cuestionario (50.0-69,9 puntos).

Los valores de la tabla 4 indican el grado de correlación encontrado entre algunas de las variables del estudio. El análisis de las tablas de contingencia entre estas variables indicó por ejemplo: que la mayor parte de los universitarios con buenos niveles de adherencia a este patrón alimentario, resultaron ser mayoritariamente normo peso; que el nivel de conocimientos previo a la intervención, resultó medio o alto en la mayoría de los individuos con normo peso y que la mayor parte de los estudiantes con nivel de conocimientos elevado, pertenecían a los EA auditivo o kinestésico.

Evaluación de la intervención educativa

Entre los aspectos mejor valorados destacan: la utilidad

TABLA 2

Distribución absoluta (n) y porcentual de los participantes en la intervención educativa según sus características demográficas, Índice de Masa Corporal (IMC), índice KidMed y estilos de aprendizaje (EA) identificados por el cuestionario VARK.

Características

Sexo

Edad

Estudios matriculados

Estado nutricional

Grado de adhesión a la DiMe

Estilos de aprendizaje preferentes

$\begin{array}{lc}\text { Femenino } & 27(41,5 \%) \\ \text { Masculino } & 38(58,5 \%) \\ \text { Mínima } & 18 \text { años } \\ \text { Máxima } & 50 \text { años } \\ \text { Media } & 22,0 \text { años } \\ \text { Fisioterapia } & 45(69,2 \%) \\ \text { Podología } & 20(30,8 \%) \\ \text { Bajo peso } & 13(20,0 \%) \\ \text { Normo peso } & 41(63,1 \%) \\ \text { Sobre peso } & 10(15,4 \%) \\ \text { Obeso } & 1(1,5 \%) \\ \text { Baja } & 15(23,1 \%) \\ \text { Media } & 30(46,1 \%) \\ \text { Alta } & 20(30,7 \%) \\ \text { Visual } & 0 \\ \text { Auditivo } & 17(26,2 \%) \\ \text { Lecto escritor } & 10(15,4 \%) \\ \text { Kinestésico } & 20(30,8 \%) \\ \text { Bimodal } & 14(21,5 \%) \\ \text { Multimodal } & 4(6,2 \%)\end{array}$

\section{TABLA 3}

Calificación media por categorías y global en las dos aplicaciones del cuestionario.

(Q1: cuestionario inicial, Q2: cuestionario posterior a las sesiones educativas, $n=$ número de encuestados).

Calificación media en Q1 y Q2 $(n=65)$

Categorías del cuestionario

I- Hábitos alimentarios

II- Estado nutricional

III- Alimentos y nutrientes

IV- Alteraciones y procesos relacionados con la alimentación Global
Q1

12,5

11,0

20,4

17,2

61,1
Q2

17,8

16,1

18,9

17,9

70,7 
de los contenidos tratados y la duración de las sesiones, considerados excelentes por más del 60.0\%. A la pregunta: "¿En general cómo evalúas la experiencia educativa?", todos la consideraron excelente. Vale la pena destacar que el 90,5\% de los universitarios reconoció tener una motivación previa muy buena o excelente y que la mayoría consideró que su asimilación de los contenidos fue buena $(30,2 \%)$, muy buena $(39,7 \%)$ o excelente $(25,4 \%)$.

\section{DISCUSIÓN}

El modelo alimentario de los participantes en el estudio (adolescentes y jóvenes universitarios), puede estar determinado por diferentes factores: preferencias alimentarias, estado de salud, poder adquisitivo, tiempo disponible para preparar las comidas, etc. (17-19). De ahí que el conocimiento de las pautas de la DiMe se considere importante pero no determinante para seguirla, esto se evidenció en los resultados que indicaron una correlación débil (Coeficiente de contingencia $V$ de Cramer=0,669; $p=0,054$ ) entre el nivel de conocimientos previos demostrados y el grado de adherencia a la DiMe.

A pesar de la gran cantidad de información referente a la nutrición y a la alimentación, que invade los medios de comunicación, los estudios que se centran en el nivel de conocimientos previos y su relación con la calidad de la dieta o el estado nutricional son escasos (20). La mayoría de los participantes demostró un nivel medio de conocimientos en nutrición y alimentación, que podría estar justificado por la procedencia de los universitarios de carreras de Ciencias de la Salud, estudios en los que es posible adquirir una base de conocimientos en nutrición y la relación de estos con el proceso salud enfermedad. No obstante, resultados de otros estudios indican que a pesar de sus conocimientos, los estudiantes universitarios suelen incurrir en conductas alimentarias poco saludables $(1,8,9,16,21)$, razón por la que se recomienda la puesta en marcha de programas de educación nutricional dirigidas a este colectivo.

La proporción de estudiantes $(30,7 \%)$ que indicó una adherencia alta a la DiMe, es similar al encontrado en universitarios de Navarra (28,4\%) (9) y Galicia (33,0\%) (1). Dado que después de la intervención educativa se detectó un aumento del número de estudiantes con nivel de conocimientos medio y alto (figura I), en el futuro sería interesante evaluar si acciones

\section{FIGURA 1}

Distribución de los estudiantes según el nivel de conocimientos demostrado en los cuestionarios inicial (Q1) y final (Q2).

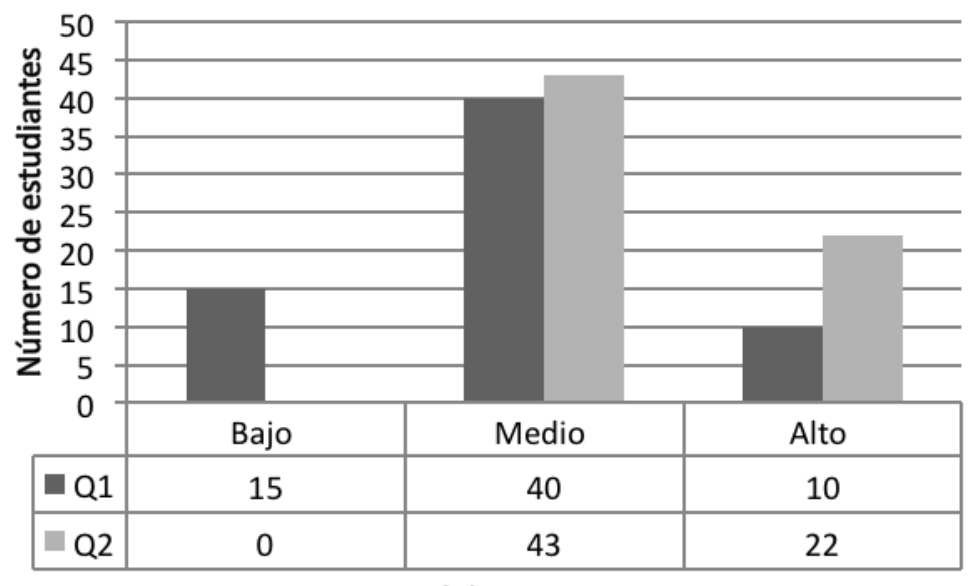

Nivel de conocimientos

TABLA 4

Resultado del análisis de correlación entre el nivel de conocimientos, estado nutricional, grado de adhesión a la DiMe, nivel de conocimientos previos y adquiridos y EA predominantemente identificados.

Variables a correlacionar

Estado nutricional y grado de adhesión a la DiMe

Nivel de los conocimientos previos y estado nutricional

Nivel de los conocimientos previos y grado de adhesión a la DiMe

Nivel de los conocimientos adquiridos y EA predominantemente identificados

$\begin{array}{cc}C C P & p \\ 0,852 & 0,022 \\ 0,701 & 0,049 \\ 0,669 & 0,054 \\ 0,693 & 0,001\end{array}$

CCP: Coeficiente de Correlación de Pearson. V de Cramer: Coeficiente de Contingencia. p: significación estadística. 
de educación nutricional similares, consiguen modificar también la calidad de la dieta y el grado de adhesión al modelo de la DiMe, que vendría a ser el objetivo a largo plazo de estas acciones educativas.

El grado de correlación hallada entre el nivel de conocimientos iniciales, respecto al estado nutricional y al grado de adherencia a la $\mathrm{DiMe}$, coincide con otros estudios que indican que los conocimientos en nutrición podrían influir en el estado nutricional (20) y en la calidad de la dieta (22). Aunque podría significar que parte de los conocimientos en nutrición que poseen los estudiantes no están basados en este modelo dietético. Es importante tener en cuenta la creciente popularidad por ejemplo de las dietas hipocalóricas, entre los adolescentes y los jóvenes; así como los resultados de estudios que sugieren que en general los jóvenes y en especial los universitarios, no tienen buenos hábitos alimentarios y consumen dietas no equilibradas (21). Una de las limitaciones del estudio es el empleo de valores de peso y altura auto referidos, para evaluar el estado nutricional, que podría introducir imprecisiones en los resultados y en las conclusiones que de ellos se extraen.

El aumento creciente del sobrepeso y la obesidad, así como la prevalencia alcanzada por los Trastornos del Comportamiento Alimentario (TCA) en diferentes países en los últimos años, es motivo de preocupación de profesionales e instituciones sanitarias. Por lo que haber detectado solo un individuo obeso, pueda considerarse como un resultado positivo. La proporción de universitarios con sobre peso (10.0\%), fue inferior a la encontrada entre universitarios de Valladolid $(27,8 \%)(8)$ y Galicia (18,6\%) (1). Al igual que en otros estudios la manifestación de bajo peso, se atribuyó fundamentalmente a las mujeres (tabla 2). Apuntando a este colectivo como un grupo de riesgo para manifestar uno de $\operatorname{los} \operatorname{TCA}(1,8,11,23)$. Teniendo en cuenta el elevado número de jóvenes que cursan estudios universitarios en España, promover acciones de educación nutricional en este colectivo podría contribuir a reducir la prevalencia del sobrepeso, la obesidad y los TCA en el futuro.

Aunque los EA se diagnosticaron solo para identificar las estrategias de aprendizaje, que podrían estar entre las preferidas por los participantes, vale la pena señalar que los estilos predominantes coinciden con los hallados en estudios anteriores en una población similar (12) y difieren parcialmente de los estilos diagnosticados entre estudiantes de medicina, en que predominó el estilo Kinestésico pero asociado al estilo Visual, no identificado entre los participantes (24).

El formato elegido para diseñar las actividades educativas (con el empleo de técnicas de enseñanza adaptadas a las estrategias de aprendizaje preferentes de la mayoría de los participantes), ha sido utilizado en otras intervenciones educativas destinadas a adolescentes y jóvenes en las también se consiguió elevar el nivel de conocimientos de los participantes (11).

\section{CONCLUSIONES}

Los universitarios participantes resultaron ser mayoritariamente normo peso y muchos de ellos deben mejorar sus hábitos alimentarios para ajustarse a las recomendaciones de la DiMe. La relación encontrada entre el nivel de conocimientos previos en nutrición y el estado nutricional, sugieren que las acciones de educación nutricional en este colectivo, podrían contribuir a mejorar la calidad de la dieta y con ella el estado nutricional y la salud futura de los universitarios.

Es recomendable completar el estudio con un análisis longitudinal, para evaluar el impacto de la intervención en el estado nutricional de los participantes y en el grado de adherencia a la DiMe.

Teniendo en cuenta que la mayor parte de los participantes evaluó la experiencia como positiva y que esta consiguió mejorar su nivel de conocimientos en nutrición y alimentación, es posible considerar la intervención educativa realizada como una herramienta eficiente en la trasmisión de conocimientos nutricionales en este colectivo.

\section{RESUMEN}

Con el objetivo de analizar la relación entre el nivel de conocimientos en alimentación, el grado de adherencia a la Dieta Mediterránea (DiMe) y el estado nutricional; se realizó un estudio de intervención con evaluación pre y post intervención; a través de tres sesiones educativas, diseñadas según las preferencias de aprendizaje de estudiantes universitarios de ciencias de la salud. Participaron 65 estudiantes de Fisioterapia y Podología, preferentemente masculinos 58,5\% y con una edad media de 22,0 años. La mayoría resultó normo peso $(63,1 \%)$, pero muchos deben mejorar sus hábitos alimentarios para ajustarse a las recomendaciones de la DiMe $(69,2 \%)$. La relación estadística encontrada entre el nivel de conocimientos previos en nutrición y el estado nutricional (Coeficiente de Correlación de Pearson $=0,7 ; p=0,049$ ), sugiere que las acciones de educación nutricional en este colectivo, podrían contribuir a mejorar la calidad de la dieta y con ella el estado nutricional y la salud futura de los universitarios. Las sesiones educativas consiguieron mejorar el nivel de conocimientos en nutrición y alimentación en la mayoría de estudiantes, por lo que es posible considerarlas como una herramienta eficiente en la trasmisión de conocimientos nutricionales en este colectivo.

Palabras clave: Hábitos alimentarios; educación nutricional; dieta mediterránea; estrategias de aprendizaje.

Nota. El proceso de diseño y validación del cuestionario de conocimientos utilizados ha sido aceptado para su publicación en la Revista Española de Nutrición Comunitaria bajo el título: Diseño y validación de un cuestionario para evaluar el nivel de conocimientos en nutrición, en estudios universitarios de Ciencias de la Salud.

\section{REFERENCIAS}

1. De la Montaña J, Castro L, Cobas N, Rodríguez M, Míguez $M$. Adherence to a mediterranean diet and its relantionship to body mass index in Galician university. Nutr Clín Diet Hosp. 2012; 32(3):72-80.

2. Serra-Majem L, Ribas L, Ngo J, Ortega RM, García A, Perez-Rodríguez C et al. Food, youth and the Mediterranean diet in Spain. Development of KidMed, Mediterranean Diet Quality Index in children and adolescent. PHN. 2004; 7(7):931-5.

3. Rodríguez Cabrero M, García Aparicio A, Salinero JJ, Pérez González B, Sánchez Fernández JJ, Gracia R et al. Diet quality and its relation to sex and BMI in adolescents. Nutr Clín Diet Hosp. 2012; 32(2):21-7.

4. Suárez MM, Ruso C, Micó A, Llopis A. Dietary pattern assesmment in Spanish adolescents in mediterranean and atlantic: a pilot study. Rev Esp Nutr Comunitaria. 2014; 20(3):85-90

5. Sofi F, Cesari F, Abbate R, Gensini GF, Casini JF. Adherence to Mediterranean diet and health status: meta-analysis. BMJ. 2008; 337:a1344.

6. Serra-Majem L, Bes-Rastrollo M, Román-Viñas B, Pfrimer K, Sánchez-Villegas A, Martínez-González MA. Dietary pat- 
terns and nutritional adequacy in a Mediterranean country. Brit J Nutr. 2009; 101(Suppl 2):S21-S28.

7. Sofi F, Abbate R, Gensini GF, Casini A. Accruing evidence on benefits of adherence to the Mediterranean diet on health: an updated systematic review and meta-analysis. Am J Clin Nutr. 2010; 92(5):1189-96.

8. Ledo-Varela MT, De Luis DA, González M, Izaola O, Conde $R$, Aller R. Nutritional characteristics and lifestyle in university students. Nutr Hosp. 2011; 26(4):814-8.

9. Durá T, Castroviejo A. Adherence to a Mediterranean diet in a college population. Nutr Hosp. 2011; 26(3):602-8.

10. García I. Education for health in the XXI century. Communication and health. Rev Esp Salud Pública. 1998; 72(2):151-3.

11. Sánchez-Socarrás V, Fornons D, Aguilar A, Vaqué C, Milà $R$. Evolution of knowledge about eating disorders through an educational intervention in post-compulsory students. Nutr Clín Diet Hosp. 2015; 35(1):32-41.

12. Sánchez-Socarrás V, Donat R, Fornons D, Vaqué $C$. Analysis of the learning styles behaviour in undergraduates and professionals health sciences. Rev Estilos de Aprendizaje. 2015; 8(1):137-61.

13. Borracci A, Manente D, Tamini S, Dvorkin M, Arribalzaga $B$, Grancelli $\mathrm{H}$ et al. Learning styles preferences from elementary school to medical postgraduate. Rev Educ Méd. 2015; 18(2):123-9.

14. Fleming D. I'm different; not dumb. Modes of presentation (VARK) in the education, proceedings of the 1995 Annual Conference of the Higher Education and Research Development Society of Australasia HERDSA. 1995; 18:308-13.

15. Serra-Majem L, Ribas L, Ngo de la Cruz J, Ortega RM, Pérez-Rodrigo C, Aranceta J. Food, younth and Mediterranean diet in Spain. Development of the KIDMED, quality index of the Mediterranean diet in childhood and adolescence. En: Serra-Majem L, Aranceta J, editores. Alimentación infantil y juvenil. Masson; (reimpresión). pp. 51-9. 2004.

16. Sánchez-Socarrás $V$, Aguilar A. Food habits and healthrelated behaviours in a university population. Nutr Hosp. 2015; 3(1):449-57.

17. Carbonell-Capella J, Esteve M, Frígola A. Snacks of chips and derived product, study market. Acceptance in a healthy diet. Rev Esp Nutr Comun. 2014; 20(3):99-108.

18. Aldaba S, Osinaga R, Urzainqui F, García-Murillo B, Falguera $E$. A healthy nutrition and exercise daily are the main cause and protection to avoid the obesity and overweight. Rev Esp Nutr Comun. 2014; 20(3):91-8.

19. Riobó P, Breton I. Intake of trans fats; situation in Spain. Nutr Hosp. 2014; 29(4):704-11.

20. Pozo A, Cubero J, Ruiz C. Previous knowledge in food and nutrition and its relationship to the body mass index of a group of students in a penitentiary centre Spanish; a resource in health education. Rev Fac Educ Albacete. 2013; 28:87-100.

21. Sánchez-Ojeda M, De Luna E. Healthy lifestyles of the university population. Nutr Hosp. 2015; 31(5):1910-9.

22. Vio F, Salinas J, Montenegro E, González CG, Lera L. Impact of a nutrition education intervention in teachers, preschool and basic school-age children in Valparaiso Region in Chile. Nutr Hosp. 2014; 29(6):1298-304.

23. Rodríguez-Cabrero M, García-Aparicio A, salinero J, PérezGonzález B, Sánchez-Fernández J, Gracia R, Robledo S, Ibáñez Moreno R. Diet quality and its relation to sex and BMI in adolescents. Nutr Clin Diet Hosp. 2012; 32(2):21-7.

24. Ponce de León-Castañeda M, Varela-Ruiz J, Lozano-Sánchez A, Ortiz-Montalvo A, Ponce-Rosas R. Profile of learning preferences of students and professors of medicine. Element to consider in teaching. Educ Med. 2010; 13(1):33-9. 